\title{
Survival prognosis and variable selection: A case study for metastatic castrate resistant prostate cancer patients
}

\section{[version 1; peer review: 2 approved]}

\author{
Søren Wengel Mogensen, Anne H. Petersen, Ann-Sophie Buchardt, \\ Niels Richard Hansen
}

Department of Mathematical Sciences, University of Copenhagen, Copenhagen, Denmark

\author{
V1 First published: 16 Nov 2016, 5:2680 \\ https://doi.org/10.12688/f1000research.8427.1 \\ Latest published: 16 Nov 2016, 5:2680 \\ https://doi.org/10.12688/f1000research.8427.1
}

\begin{abstract}
Survival prognosis is challenging, and accurate prediction of individual survival times is often very difficult. Better statistical methodology and more data can help improve the prognostic models, but it is important that methods and data usages are evaluated properly. The Prostate Cancer DREAM Challenge offered a framework for training and blinded validation of prognostic models using a large and rich dataset on patients diagnosed with metastatic castrate resistant prostate cancer. Using the Prostate Cancer DREAM Challenge data we investigated and compared an array of methods combining imputation techniques of missing values for prognostic variables with tree-based and lasso-based variable selection and model fitting methods. The benchmark metric used was integrated AUC (iAUC), and all methods were benchmarked using cross-validation on the training data as well as via the blinded validation. We found that survival forests without prior variable selection achieved the best overall performance ( $\mathrm{cV}$-iAUC $=0.70$, validation-iACU $=0.78)$, while a generalized additive model was best among those methods that used explicit prior variable selection ( $\mathrm{CV}$-iAUC $=0.69$, validation-iACU $=0.76$ ). Our findings largely concurred with previous results in terms of the choice of important prognostic variables, though we did not find the level of prostate specific antigen to have prognostic value given the other variables included in the data.
\end{abstract}

\section{Keywords}

imputation, lasso , generalized additive models, stability selection , survival forests, survival prognostic models

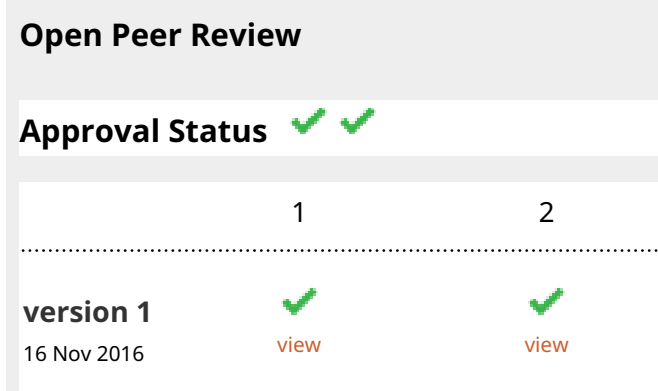

1. Sarah Lemler, Ecole Centrale Supélec,

Châtenay-Malabry, France

2. Jacob von Bornemann Hjelmborg (iD), University of Southern Denmark, Odense,

Denmark

Any reports and responses or comments on the article can be found at the end of the article. 
Corresponding author: Niels Richard Hansen (niels.r.hansen@math.ku.dk)

Competing interests: No competing interests were disclosed.

Grant information: The author(s) declared that no grants were involved in supporting this work.

Copyright: ( 2016 Wengel Mogensen S et al. This is an open access article distributed under the terms of the Creative Commons Attribution License, which permits unrestricted use, distribution, and reproduction in any medium, provided the original work is properly cited.

How to cite this article: Wengel Mogensen S, H. Petersen A, Buchardt AS and Hansen NR. Survival prognosis and variable selection: A case study for metastatic castrate resistant prostate cancer patients [version 1; peer review: 2 approved] F1000Research 2016, 5:2680 https://doi.org/10.12688/f1000research.8427.1

First published: 16 Nov 2016, 5:2680 https://doi.org/10.12688/f1000research.8427.1 


\section{Introduction}

The Prostate Cancer DREAM Challenge ${ }^{1,2}$, launched March 16, 2015, was a prediction competition, which challenged the participating teams to develop better prognostic models for survival of patients with metastatic castrate resistant prostate cancer. The full competition was divided into two subchallenges, with subchallenge $1 \mathrm{a}$ and $1 \mathrm{~b}$ on prediction of survival and subchallenge 2 on prediction of discontinuation of treatment. Three of the authors of this paper participated in the challenge (as part of team KUStat) and made a final submission for subchallenge 1 . We report here our findings and methodology developed for subchallenge 1a as well as in subsequent work carried out after the final submission.

The Prostate Cancer DREAM Challenge offered a large and complex dataset from four clinical trials containing data for around 2000 patients and with more than 100 potential predictor variables. The participating teams were free to develop any model, but predictions - in terms of risk scores - were assessed and compared in a fixed framework via submissions of predictions through a web interface. For the assessment data used, the survival status was held back from the participants, but the assessment system was fully disclosed, and we as participants could mimic the assessment procedure on the released data to optimize predictive performance.

A reference prognostic model existed ${ }^{3}$ when the challenge was launched, and it was a requirement that the top-performing team could demonstrate an improvement over this reference model. The main scoring metric for assessing the prognostic models was time integrated AUC (iAUC). Halabi and coauthors ${ }^{3}$ reported iAUC estimates of 0.73 and 0.76 for the reference model on a test and validation dataset, respectively.

The Prostate Cancer DREAM Challenge included three rounds of submissions to a leaderboard prior to the final submission, and we submitted predictions for the second round of the leaderboard, which achieved an iAUC of 0.8062 . This appeared to be a clear improvement of the reference model, which achieved an iAUC of 0.7782 in the same leaderboard round. Our final submission achieved an iAUC of 0.7732 , which placed our team roughly in the middle of a big group of 15 teams that achieved an iAUC between 0.77 and 0.78 and well above the reference model, which achieved an iAUC of 0.7429 in the final scoring. However, the winning team managed to distinguish itself from the rest with an iAUC of 0.7915 .

Our submission was based on a variable selection method called stability selection and a subsequent fit of a generalized additive model. Some ad hoc modifications were made, but it was unclear if they had any positive effect on the predictive strength of the model. We also experimented with different techniques for imputation as there are a large number of missing values in the dataset for some variables. The effect of the imputation technique was, however, not fully understood, though we suspected that more sophisticated imputation techniques had a negative effect on predictive performance.

In this paper, we report a systematic evaluation of a total of 24 combinations of methods for model fitting, variable selection and imputation. These include the methods we used for our participation in the Prostate Cancer DREAM Challenge, some methods that we tried, but found inferior, and some additional methods that we afterwards found could potentially improve on the generalized additive model. The paper is organized as follows: first we present some descriptive and exploratory aspects of the dataset, and we describe how the dataset was prepared for the model building and evaluation process; then we briefly describe all the different methods we considered, the $\mathrm{R}$ functions and packages that implement the methods used and the analysis pipeline; finally, we present our results and conclusions.

\section{Data}

The Prostate Cancer DREAM Challenge dataset comprises patient baseline data as well as extensive longitudinal data tables from the comparator arm of four clinical trials: ASCENT-2 ${ }^{4}$, MAINSAIL ${ }^{5}$, $\mathrm{VENICE}^{6}$, and ENTHUSE-33 ${ }^{7}$. We will in this paper only consider the use of baseline variables for survival prognosis. Data from three of the four trials was released as training data for the Prostate Cancer DREAM Challenge, see Table 1, which includes followup survival and treatment discontinuation information. Data from the fourth trial (ENTHUSE-33, 470 patients) was released for leaderboard (157 patients) and final scoring (313 patients), and did not include followup survival information. The latter dataset comprising the 313 patients from the ENTHUSE-33 trial will be referred to as the validation data. Though we have not had access to survival times for the validation data, predictions for the validation data could be assessed via the Prostate Cancer DREAM Challenge web interface.

We note that the survival distributions for the three trials in the training data are comparable, see Figure 1 in 2 (the $p$-value for the log-rank test of equal survival functions is 0.63 ), but we also note that the followup time for the VENICE trial was considerably longer than for the other two trials.

To assess prognostic models, it is important to understand the target population. The patients included in the four trials were not necessarily recruited from the same population, and Table 2, Table 3 , and Table 4 present breakdowns of the patients in the four trials according to age group, geography, and race, respectively. We note that the age distribution for the ASCENT-2 trial differs from the other three trials with a larger proportion of patients over 75 years old and a smaller proportion in the group 18-64 years. The age distributions for the other three trials are similar. We also note that the majority of patients are white and from Europe or North

\section{Table 1. Number of patients and registered deaths from the three clinical trials in the training data.}

\begin{tabular}{|r|r|r|}
\hline Trial & Nr. of patients & Nr. of deaths \\
\hline ASCENT-2 & 476 & 138 \\
\hline MAINSAIL & 526 & 92 \\
\hline VENICE & 598 & 433 \\
\hline Total & 1600 & 663 \\
\hline
\end{tabular}


Table 2. Number of patients stratified according to age group and trial.

\begin{tabular}{|r|r|r|r|r|r|}
\hline & ASC.-2 & MAINSAIL & VENICE & ENT.-33 & Total \\
\hline $18-64$ & 111 & 171 & 219 & 111 & 612 \\
\hline $65-74$ & 211 & 246 & 254 & 141 & 852 \\
\hline$\geq 75$ & 154 & 109 & 125 & 61 & 449 \\
\hline Total & 476 & 526 & 598 & 313 & 1913 \\
\hline
\end{tabular}

Table 3. Number of patients stratified according to geographic region and trial.

\begin{tabular}{|r|r|r|r|r|r|}
\hline ASC.-2 & MAINSAIL & VENICE & ENT.-33 & Total \\
\hline W. Europe & 0 & 247 & 212 & 104 & 563 \\
\hline N. America & 0 & 139 & 80 & 61 & 280 \\
\hline E. Europe & 0 & 84 & 127 & 50 & 261 \\
\hline S. America & 0 & 0 & 86 & 38 & 124 \\
\hline Asia/Pacific & 0 & 0 & 0 & 47 & 47 \\
\hline Africa & 0 & 0 & 0 & 13 & 13 \\
\hline Other & 0 & 50 & 93 & 0 & 143 \\
\hline Missing & 476 & 6 & 0 & 0 & 482 \\
\hline Total & 476 & 526 & 598 & 313 & 1913 \\
\hline
\end{tabular}

Table 4. Number of patients stratified according to race and trial.

\begin{tabular}{|r|r|r|r|r|r|}
\hline ASC.-2 & MAINSAIL & VENICE & ENT.-33 & Total \\
\hline Asian & 419 & 433 & 538 & 225 & 1615 \\
\hline Black & 5 & 0 & 36 & 49 & 90 \\
\hline Hispanic & 14 & 25 & 17 & 12 & 86 \\
\hline Other & 6 & 0 & 0 & 0 & 14 \\
\hline Missing & 0 & 13 & 7 & 27 & 53 \\
\hline Total & 476 & 55 & 0 & 0 & 55 \\
\hline
\end{tabular}

America. For the ASCENT-2 trial there is no geographic data, but it is known that these patients were recruited from North America and Europe ${ }^{4}$. The ASCENT-2 trial is, furthermore, the only trial for which the ethnicity group "Hispanic" is registered as race. A notable difference between the trials is that the validation data from the ENTHUSE-33 trial contains a larger proportion of Asians, and there are apparently no patients from the Asian/Pacific region in the training data. A further breakdown of the geographic group "Other" shows that the majority of these patients are white, but 30 patients from the VENICE trial are Asians; therefore they could be from the Asian/Pacific region. Nevertheless, there is little variability in the data in terms of race and geographic region.

All the baseline values for the predictor variables were made available for the Prostate Cancer DREAM Challenge participants in a data table referred to as CoreTable. The variables in CoreTable were filtered and cleaned prior to the model building. The filtering consisted of excluding variables that were mostly or entirely missing in either the training or the validation data, or had no variation. Variables related to treatment and discontinuation were excluded as well. The cleaning consisted of consistent registration of missing values, correct registration of variable type (categorical or numeric), and some recoding. The filtered and cleaned data contains 93 predictor variables in addition to the followup survival time, the death indicator, and the patient ID, see Table S1. Of the 93 predictors 72 are binary predictors, 4 are categorical predictors with three or more categories, and 20 are numerical predictors, which, except for BMI, are laboratory measurements. Table S1 shows that there are a considerable number of missing values in the training data for some of the numerical variables, while the validation data set is more complete. In fact, 37 of the 63 missing values in the validation data are related to only two patients, for whom most laboratory measurements are missing.

Figure 1 shows pairwise correlations between binary predictors and correlations and associations between the numerical predictors. The variables were ordered by hierarchical clustering based on the correlations. We note that there is some correlation among the predictors. Among the numerical predictors, the variables $C A$, ALB, HB and NA. are positively correlated and also correlated with total protein TPRO. The variables NEU (neutrophils, a white blood cell type) and WBC (white blood cells) are unsurprisingly strongly positively correlated, and they are both positively correlated with PLT (platelets). Finally, the group PSA, ALP, AST, ALT and LDH also shows positive correlations with AST (aspartate aminotransferase) and ALT (alanine transaminase) being strongly correlated. For the binary variables, it is worth noting that the correlation pattern is rather weak and does not cluster in any clear pattern, though there is a certain weak overall positive correlation pattern. We see this pattern most clearly for the variables related to medical history (see Supplementary Figure 1 at the end of the paper for labels with medical history variables having prefix $\mathrm{MH}$ ). Some of the strongest clustered correlations are unsurprising, such as the positive correlation among MI, MHCARD and BETA_BLOCKING related to cardiac disorders, the positive correlation between the metabolism and diabetes variables MHMETAB and DIAB, and the negative correlation between GONADOTROPIN (fertility medication) and ORCHIDECTOMY (testicle(s) removed).

\section{Methods}

Imputation

As mentioned above, the training data contains a large number of missing values. To deal with the missing values we implemented three imputation schemes: imputation under the missing completely at random assumption (MCAR), imputation under the missing at random assumption using only other predictors (MAR), and imputation under the missing at random assumption using other predictors as well as the survival response (MARwR). The variable to be imputed is denoted VI in the following.

The MCAR assumption means, as the name suggests, that the mechanism resulting in missing values is completely random and independent of both observed and unobserved variables. The corresponding imputation scheme is implemented by drawing 

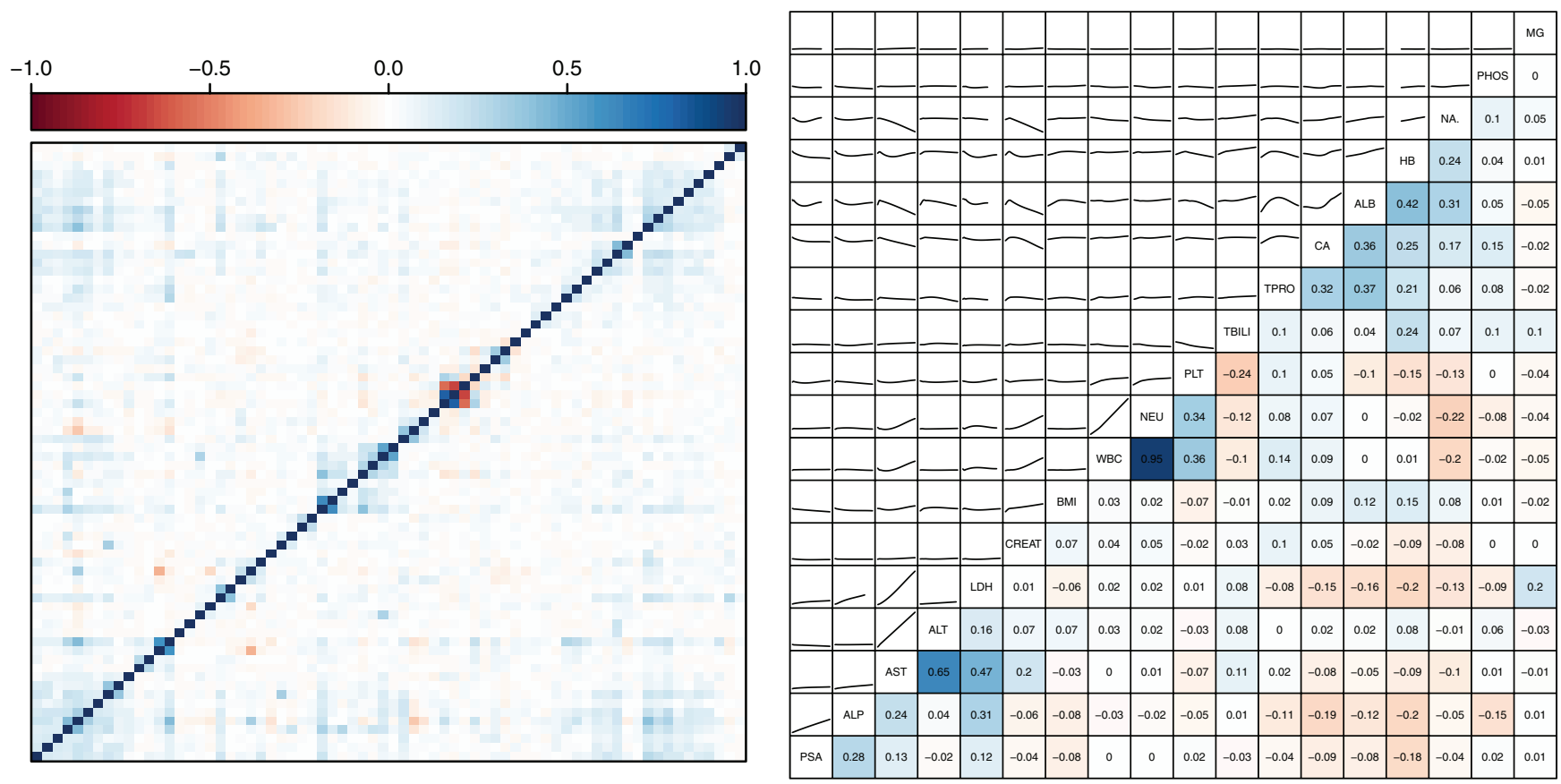

Figure 1. Correlation plot (left) for all binary predictors. See Supplementary Figure 1 for the correlation plot with labels. Correlations (right, below the diagonal) and pairwise associations as given by loess scatter plot smoothers (right, above the diagonal) for the numerical predictors.

observations randomly from the empirical marginal distribution of VI.

The two other imputation schemes involve fitting regression models with VI as the response variable, and their implementations share a number of components. Both schemes can use up to five other variables from the dataset to fit a linear regression model of VI. The five predictors are selected as the variables having the strongest marginal association with VI measured in terms of $p$-values. Only variables with $p \leq 0.05$ and no missing values are considered. Missing values of VI are then imputed from the fitted regression model. The MAR scheme uses only predictor variables whereas the MARwR uses the censored survival times as well. Our MARwR scheme follows the suggestions by White and Royston ${ }^{8}$ to use the Nelson-Aalen estimate of the cumulative hazard function together with the indicator variable for censoring in the imputation model of VI.

We did not implement a specific MAR or MARwR scheme for categorical variables, and the missing values of RACE $C$ and REGION $C$ were therefore imputed using the MCAR scheme.

\section{Proportional hazards models}

All methods considered except random survival forests are based on the proportional hazards model with the hazard function for the $i$ th patient being

$$
\lambda_{i}(t)=e^{f\left(\mathbf{x}_{i}\right)} \lambda_{0}(t)
$$

Here $\lambda_{0}$ is a baseline hazard function and $f$ is a function of the vector of predictor variables, $\mathbf{x}_{i}=\left(x_{i j}\right)_{j}$, for the $i$ th patient. We refer to $f\left(\mathbf{x}_{i}\right)$ as the risk score for the $i$ th patient. For the purpose of risk prediction in the context of the Prostate Cancer DREAM Challenge, any monotonically increasing transformation of $f\left(\mathbf{x}_{i}\right)-$ e.g. $\exp \left(f\left(\mathbf{x}_{i}\right)\right)-$ is an equivalent risk score.

The additive, linear model is given by

$$
f\left(\mathbf{x}_{i}\right)=\sum_{j=1}^{p} \beta_{j} x_{i j} .
$$

The coefficients $\beta_{j}$ can be estimated by maximizing Cox's partial likelihood using the function coxph from the survival $R$ package $^{9,10}$. However, for a large number of predictors there will usually be a favorable bias-variance tradeoff by using shrinkage and/or variable selection. Moreover, the additive, linear model may not be adequate, since it does not capture nonlinear or interaction effects on the log-hazard scale.

The generalized additive model is given by

$$
f\left(\mathbf{x}_{i}\right)=\sum_{j=1}^{p} f_{j}\left(x_{i j}\right),
$$

for functions $f_{j}$ of the univariate predictors. For numerical predictors the functions $f_{j}$ are generally assumed to be smooth. The model can be fitted to data by minimizing the negative logpartial-likelihood with a quadratic penalty that penalizes roughness of the $f_{j}$-functions. This can be achieved by the function gam with family $=\operatorname{cox} \cdot \mathrm{ph}()$ from the mgcV R package ${ }^{11}$. The function gam automatically chooses the trade-off between likelihood and 
penalty (and hence the smoothness) via built-in optimization of an unbiased risk estimate.

\section{Lasso}

Lasso is a shrinkage and selection estimator that fits a proportional hazards model by minimizing the negative log-partial-likelihood with an $\ell_{1}$-penalty. The lasso estimator can be computed using the function glmnet with family $=$ "cox" from the glmnet $R$ package $^{12,13}$. It fits models for a sequence of penalty parameters (the lasso path), and it supports selection of the penalty parameter via built-in cross-validation. Any choice of the penalty parameter will generally lead to some coefficients shrunk to 0 , which can be interpreted as a variable selection procedure. For all the results presented in this paper, the penalty parameter for lasso was chosen by minimizing the cross-validated partial likelihood loss.

Lasso, with the penalty chosen as describe above, yields an additive, linear model and gives resulting estimates of the risk score. Some coefficients are shrunk to 0 , hence lasso does implicit variable selection, but the coefficients for the selected variables are, in addition, shrunk toward 0 . The debiased lasso re-estimates the coefficients for the lasso selected variables without shrinkage, and can be computed by coxph based on the variables selected by lasso.

\section{Stability selection}

Stability selection ${ }^{14}$ is a variable selection method that works by choosing variables that are stably selected on subsampled data by e.g. lasso. The method implemented is a slight adaptation of the method proposed by Meinshausen and Bühlmann in 14, which works as follows: The lasso path is computed for a subsample of the training data, cross-validation is used on the subsample to select the optimal penalty, and the coefficients not shrunk to 0 are selected for the subsample. To obtain the results reported in this paper we used the procedure with 100 replications and with each subsample being half the size of the full training data. The selection frequency was computed for all variables, and a cutoff for stably selected variables was chosen to be $50 \%$.

Any method for fitting a survival regression model can be combined with stability selection by fitting the model using only the stably selected variables.

\section{Stochastic gradient boosting}

A gradient boosting machine fits base learners sequentially to socalled pseudo-residuals. A base learner is a simple model of $f$, e.g. one small regression tree, and the ensemble estimate of $f$ consists of an aggregate of all the base learners. Regularization by shrinkage may be applied for each base learner. A stochastic gradient boosting machine samples (without replacement) for each iteration a subset of observations uniformly from the training data and uses only this subset for fitting a particular base learner ${ }^{15}$.

We used an implementation of a stochastic gradient boosting machine with trees as base learners that directly optimizes a smoothed version of the concordance index (C-index) as described in 16. The implementation is available on GitHub ${ }^{17}$, which is a fork of an earlier version of the gbm $R$ package ${ }^{18}$. This implementation implicitly applies shrinkage when fitting an individual tree, as an optimal solution is not guaranteed ${ }^{16}$. Pilot experiments indicated that additional explicit shrinkage did not improve the predictions, and therefore our implementation does not use explicit shrinkage. The subsampling fraction (bag fraction) controls the number of observations used for each tree fit. Our implementation sets the subsampling fraction to 0.5 , allows for interactions of up to three variables, and uses a minimum node size of 10 . The number of trees is chosen by built-in cross-validation with a maximum of 1000.

We fitted gradient boosting machines using all 93 predictor variables in the dataset as well as using only the stably selected variables.

\section{Random survival forests}

A random survival forest is an ensemble method similar to a boosting machine that uses trees as base learners ${ }^{19}$. For each iteration of the algorithm, a dataset of the same size as the original is sampled with replacement. A tree is then grown using this data set. For each node of the tree, a subset of variables is sampled and considered for splitting. The splitting is done according to one of the variables in order to maximize survival difference as measured by the log-rank test statistic. In each terminal node, a Nelson-Aalen estimate is calculated and the estimates are then aggregated into an ensemble fit of the cumulative hazard function. To obtain a single predicted outcome for each subject, we used ensemble mortality as defined in 19.

Random survival forests can be fitted using the randomForestSRC R package $^{19}$. Our implementation uses 1000 trees with a minimum node size of 6 (number of events in terminal nodes). For each split the procedure considers 20 candidate variables, and for each of those variables a maximum of 10 potential splitting points are randomly chosen. Setting a maximum of potential splitting points has two purposes. First, it speeds up computations. Second, it counters the fact that the algorithm is biased towards splitting on continuous variables as opposed to variables with only a few levels ${ }^{20}$.

As for stochastic gradient boosting, survival forests were fitted using all predictor variables as well as only the stably selected variables.

\section{The score metric}

Survival prognosis can be viewed as a prediction of a binary variable (is the patient dead) at each future time point, in which case the prognosis by the risk score can be evaluated using the conventional AUC score at any given time point. The time integrated AUC (iAUC) constitutes a single summary score, and it was the main score metric for the DREAM subchallenge 1a. The score can be estimated using the timeROC R package ${ }^{21}$.

\section{The modeling and assessment pipeline}

The methods described above for imputation, for fitting a survival model, and for variable selection can be combined in a number of ways. We implemented all meaningful combinations resulting in a total of 24 prognostic models, see Table 5. 
Table 5. The eight used combinations of variable selection and methods for fitting a survival model. All eight combinations were used in combination with all the three imputation methods: MCAR, MAR and MARwR.

\begin{tabular}{|l|c|c|}
\hline Method & All variables & $\begin{array}{c}\text { Stab. selected } \\
\text { variables }\end{array}$ \\
\hline Lasso & $\checkmark$ & \\
\hline $\begin{array}{l}\text { Debias. } \\
\text { Lasso }\end{array}$ & $\checkmark$ & \\
\hline Cox & & $\checkmark$ \\
\hline Gam & & $\checkmark$ \\
\hline Forest & $\checkmark$ & $\checkmark$ \\
\hline Boosting & $\checkmark$ & $\checkmark$ \\
\hline
\end{tabular}

The final submission for the Prostate Cancer DREAM challenge from team KUStat was based on a generalized additive model using stability selected variables and the MCAR imputation scheme. Some hand tuning of the final submission was made, see our write-up ${ }^{22}$ for details. The hand tuning was not implemented for this paper.

The implementation consists of a collection of supporting $\mathrm{R}$ functions and a main training and prediction function that fits all the 24 models on a training data set and returns the risk scores for a test data set. The assessment pipeline consists of a 5-fold crossvalidation step to estimate iAUC using the training data only, and a refit step where the models are fitted to the full training dataset and risk scores are predicted for the validation dataset.
For the iAUC estimates reported in this paper, we replicated the 5-fold cross-validation 3 times and averaged the results to decrease the variation due to the random data splits. For the iAUC estimates based on the validation data we submitted predictions to the post-challenge leaderboard to assess the predictions on the 313 patients from the ENTHUSE-33 trial. We made two submissions for each model and averaged the results. Though we only found minor variations in the results for the two submissions, the double submission was done because several aspects of the model fitting rely on randomness. We wanted the results to be robust to this variation.

\section{Results}

Figure 2 shows the iAUC score as estimated by cross-validation and on the validation data for all 24 combinations of methods. First, we observe that, in general, the iAUC was lower when the response was used for imputation (MARwR). The two other imputation schemes gave comparable results, and the results reported below refer to MCAR as well as MAR imputation in combination. Survival forests were overall best with iAUC around 0.78 on the validation data and just below 0.70 in the cross-validation. Debiased lasso was worst with an iAUC around 0.73 on the validation data and 0.66 in the cross-validation. The differences are small, and we also note a large variation between folds in the cross-validation indicating heterogeneity in the training data.

The generalized additive model was the best among those that relied on stability selection with iAUC around 0.76 on the validation data and 0.69 in the cross-validation. The pure lasso prediction did surprisingly well on the validation data, compared to the
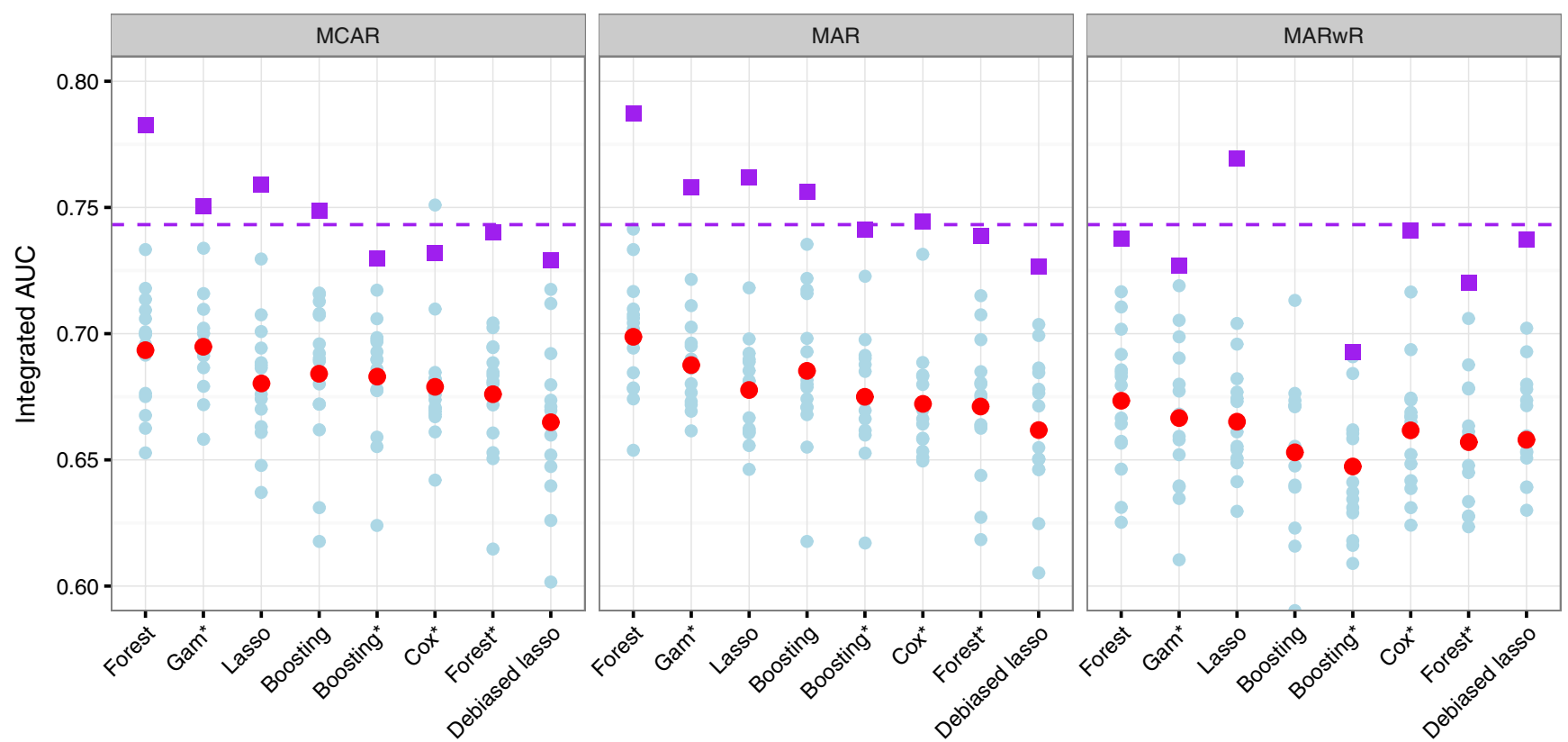

Method

Figure 2. Integrated AUC for different combinations of methods evaluated by three replications of $\mathbf{5}$-fold cross-validation. Results are shown for individual folds (light blue filled circles) and averaged over all folds (red filled circles). The figure also shows iAUC on the validation data (purple filled squares) and iAUC for the reference model on the validation data (purple dashed line). The four methods marked with a * used variables chosen via stability selection, whereas the other four methods relied on implicit variable selection. 
cross-validation results and irrespectively of the imputation method, and the computationally much more expensive boosting method was only just on par with lasso overall.

The results from stability selection are interesting in themselves. Figure 3 shows selection proportions for the 20 most often selected predictors for each of the imputation methods. These results are from one run of the algorithm with 100 subsamples. The variability due to the random subsampling was found to be small, though some variables would cross the (somewhat arbitrary) threshold of 50\% in some runs and not in others. Figure 3 is from one of the two replications used for the validation.

The eight variables AST, HB, ALP, ECOG C, ADRENAL, LIVER, ANALGESICS and ALB were selected in a large proportion of the subsamples, irrespective of the imputation scheme. The variable RACE_C just reached the 50\% threshold for all three imputation schemes, while BMI, ACE_INHIBITORS and ESTROGENS reached the 50\% threshold for some, but not all, imputation schemes. Notably, PSA was not stably selected. It is also noteworthy that ALB (which has 493 missing values) increased its selection proportion considerably when imputed using the response.

\section{Discussion}

It is difficult to correctly evaluate how well a prognostic model will generalize. We believe that competitions like the Prostate Cancer DREAM Challenge have a positive effect on the development of systematic approaches to model evaluation. However, the big differences between the cross-validated estimates of iAUC and those obtained on the validation data demonstrate how difficult it is to generalize from one dataset to another. Such differences in predictive strength, as measured by iAUC, can be explained by differences in either the composition of the patients, or in how their survival is related to the predictors, but we cannot offer a more detailed explanation. In addition, we interpret the large variation between the cross-validation folds as evidence of a heterogeneous training dataset. This is not surprising, given that the training data is pooled from three different trials. Moreover, we noted that the majority of patients in the dataset are white and from Europe or North America. Thus it is also difficult to tell how well a prognostic model based on the Prostate Cancer DREAM Challenge data will generalize to other populations.

On the other hand, even if the absolute values of iAUC are incomparable, the rankings of the fitted models obtained by either cross-validation or validation were roughly the same. Thus we believe that our results shed light on which methods are most useful for developing prognostic survival models and for selecting variables of prognostic value.

For variable selection we believe that the poor performance of debiased lasso is a consequence of lasso generally selecting too many variables - see Chapter 8 in 23 for an extensive treatment of variable selection with lasso - and thus without shrinkage of the corresponding coefficients, debiased lasso will overfit. Stability
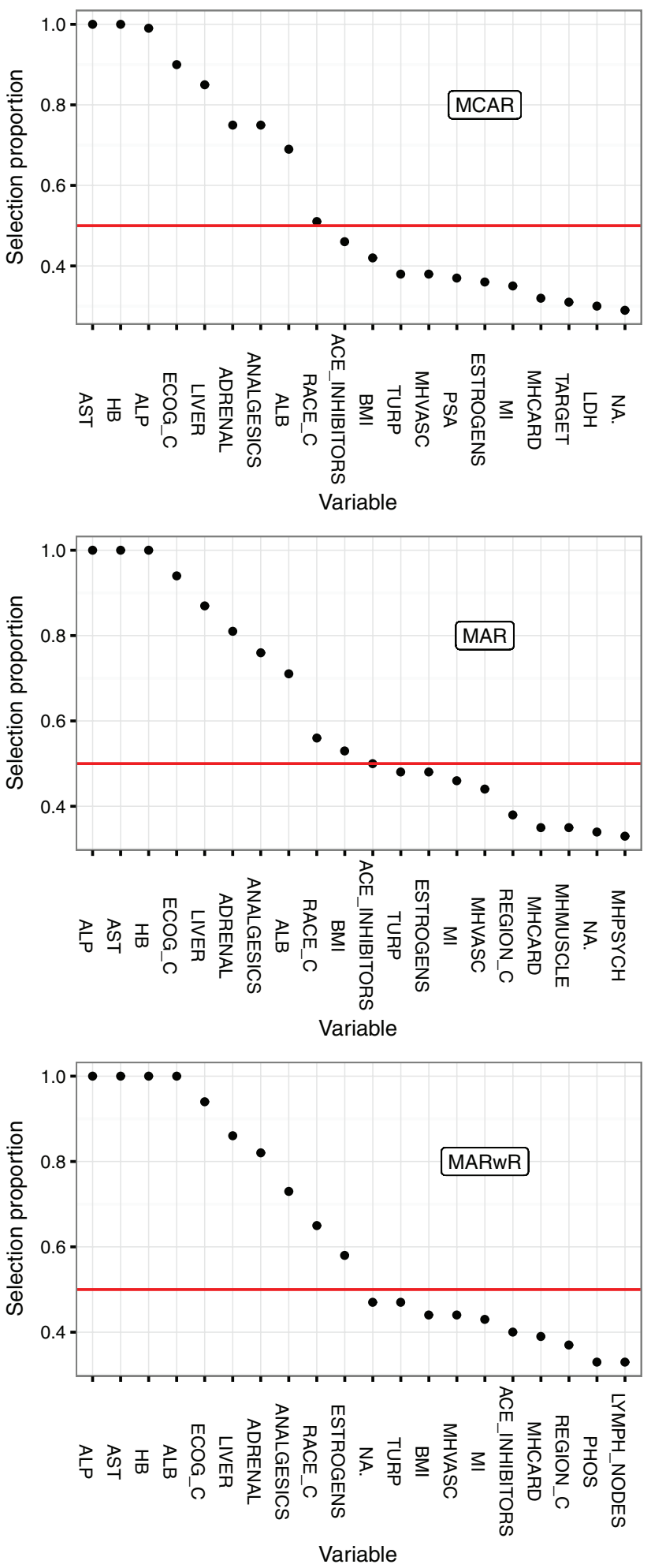

Figure 3. Selection proportions for the $\mathbf{2 0}$ most stably selected variables stratified by imputation method. The threshold of $50 \%$ (red line) was used for the final variable selection. 
selection is a more stringent selection criterion, which is less prone to select false positives, see Chapter 10 in 23 and 14. The variables selected by stability selection as having prognostic value also largely agree with those found in 3 and used in the reference model. One difference is that the DREAM dataset gives nuanced information on disease sites, and we found that liver and adrenal lesions, in particular, had prognostic value. The PSA variable was, on the other hand, not selected. It was a predictor in the reference model, but not a very strong one. Based on this study we therefore recommend stability selection as a reliable method for selecting prognostic variables.

For imputation of missing values the use of the response seemed to degrade predictive performance. This contradicts the recommendations in e.g. 8, which presents a simulation study showing that imputation based on inclusion of an estimate of the cumulative hazard function and the indicator of censoring is superior to a number of other imputation schemes. The framework of 8 is, however, focused on parameter estimation and hypothesis testing using multiple imputation, where the objectives differ from those of prognostic modeling. We believe that further investigations into the effect of imputation - in particular when using the response - are needed to fully understand benefits and pitfalls, but our recommendations based on this study is to avoid using the response for imputation when building prognostic models.

Finally, the best performing model - the survival forest - is the only model considered that is not based directly on the proportional hazards assumption. Thus we may speculate that this assumption could be violated.

\section{Conclusions}

Survival forests without explicit variable selection gave the best performance overall in the cross-validation and on the validation data. When stability selection was used for explicit variable selection, the generalized additive model gave the best performance. Imputation using the response appeared to have a negative effect on predictive performance.

The four stably selected laboratory measurements AST, HB, ALP and ALB and the ECOG performance status were selected as some of the most important prognostic factors, together with liver and adrenal lesions and prior use of analgesics.

\section{Data and software availability}

The Challenge datasets can be accessed at: https://www.projectdatasphere.org/projectdatasphere/html/pcdc
Challenge documentation, including the detailed description of the Challenge design, overall results, scoring scripts, and the clinical trials data dictionary can be found at: https://www.synapse.org/ ProstateCancerChallenge

The code and documentation underlying team KUStat's challenge submission can be found at: http://dx.doi.org/10.7303/ syn $4260742^{22}$

The $\mathrm{R}$ code and documentation underlying the methods presented in this paper can be found at: https://github.com/nielsrhansen/ProstateDream. An archived version as at the time of publication is available at: http://dx.doi.org/10.5281/zenodo.50872 24

\section{Author contributions}

NRH initiated the participation in the Prostate Cancer DREAM Challenge as a component in the Master's program in Statistics at University of Copenhagen for SWM, AHP and ASB. AHP contributed with methods for imputation, ASB contributed with exploratory analysis and stability selection using lasso, SWM contributed with survival forests and gradient boosting and NRH contributed with generalized additive models and stability selection. All authors contributed to the integration of the methods into a complete analysis pipeline. NRH wrote the first draft of the manuscript and all authors contributed to its finalization.

\section{Competing interests}

No competing interests were disclosed.

\section{Grant information}

The author(s) declared that no grants were involved in supporting this work.

\section{Acknowledgements}

This publication is based on research using information obtained from www.projectdatasphere.org, which is maintained by Project Data Sphere, LLC. Neither Project Data Sphere, LLC nor the owner(s) of any information from the web site have contributed to, approved or are in any way responsible for the contents of this publication. The authors acknowledge Maria Bekker-Nielsen Dunbar and Lisbeth Tomaziu, who worked on subchallenge 2 as part of the KUStat team participating in the Prostate Cancer DREAM Challenge. The authors also acknowledge Jing Zhou, who worked with the KUStat team but made her own submission to the Prostate Cancer DREAM Challenge. Finally, the authors acknowledge Sage Bionetworks, the DREAM organization, and Project Data Sphere for developing and supplying data for the challenge. 


\section{Supplementary material}

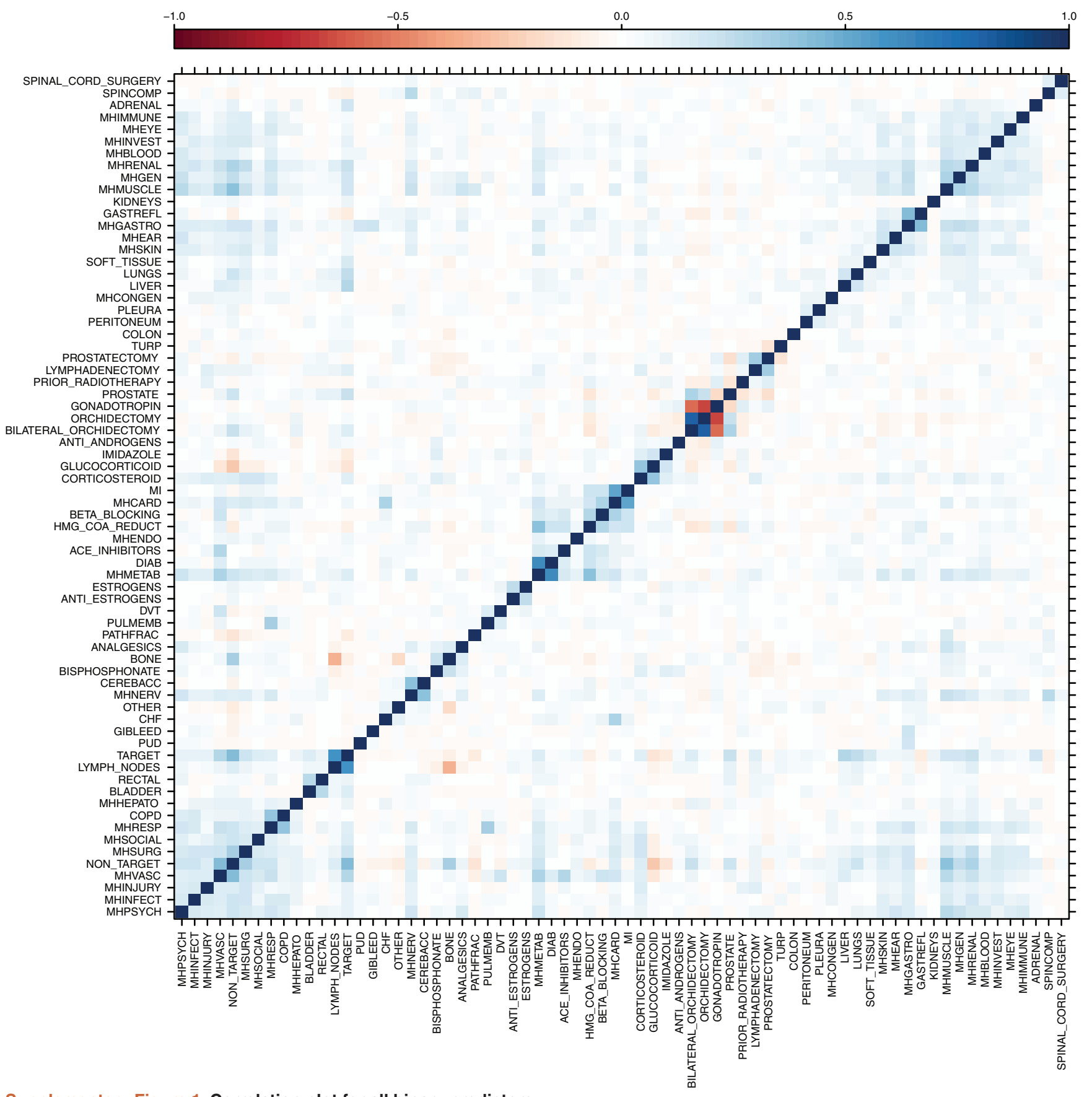

Supplementary Figure 1. Correlation plot for all binary predictors. 
Table S1. Descriptions of the 93 predictor variables included in the modeling. The variables selected by stability selection by any of the imputation methods are marked with a *

\begin{tabular}{|c|c|c|c|c|}
\hline Variable name & Type & Description & $\begin{array}{l}\text { Missing } \\
\text { in training }\end{array}$ & $\begin{array}{l}\text { Missing in } \\
\text { validation }\end{array}$ \\
\hline AGEGRP2 & Cat. (ord) & Age Group (3 categories) & 0 & 0 \\
\hline RACE_C* & Cat. & Race (5 categories) & 55 & 0 \\
\hline REGION_C & Cat. & Region of the World (5 categories) & 482 & 0 \\
\hline ECOG_C* & Cat. (ord) & Baseline Patient Performance Status & 1 & 0 \\
\hline NON_TARGET & Binary & Baseline Non-Target Lesion(s) & 0 & 0 \\
\hline TARGET & Binary & Baseline Target Lesion(s) & 0 & 0 \\
\hline BONE & Binary & Baseline Bone Lesion(s) & 0 & 0 \\
\hline RECTAL & Binary & Baseline Rectal Lesion(s) & 0 & 0 \\
\hline LYMPH_NODES & Binary & Baseline Lymph Node Lesion(s) & 0 & 0 \\
\hline KIDNEYS & Binary & Baseline Kidney Lesion(s) & 0 & 0 \\
\hline LUNGS & Binary & Baseline Lung Lesion(s) & 0 & 0 \\
\hline LIVER* $^{*}$ & Binary & Baseline Liver Lesion(s) & 0 & 0 \\
\hline PLEURA & Binary & Baseline Pleura Lesion(s) & 0 & 0 \\
\hline OTHER & Binary & Baseline Other Lesion(s) & 0 & 0 \\
\hline PROSTATE & Binary & Baseline Prostate Lesion(s) & 0 & 0 \\
\hline ADRENAL* & Binary & Baseline Adrenal Lesion(s) & 0 & 0 \\
\hline BLADDER & Binary & Baseline Bladder Lesion(s) & 0 & 0 \\
\hline PERITONEUM & Binary & Baseline Peritoneum Lesion(s) & 0 & 0 \\
\hline COLON & Binary & Baseline Colon Lesion(s) & 0 & 0 \\
\hline SOFT_TISSUE & Binary & Baseline Soft Tissue Lesion(s) & 0 & 0 \\
\hline ORCHIDECTOMY & Binary & Prior Orchidectomy(includes bilateral) & 0 & 0 \\
\hline PROSTATECTOMY & Binary & Prior Prostatectomy & 0 & 0 \\
\hline TURP & Binary & Prior Turp & 0 & 0 \\
\hline LYMPHADENECTOMY & Binary & Prior Bilateral Lymphadenectomy & 0 & 0 \\
\hline SPINAL_CORD_SURGERY & Binary & Prior Spinal Cord Surgery & 0 & 0 \\
\hline BILATERAL_ORCHIDECTOMY & Binary & Prior Bilateral Orchidectomy & 0 & 0 \\
\hline PRIOR_RADIOTHERAPY & Binary & Prior Radiotherapy & 0 & 0 \\
\hline ANALGESICS* & Binary & Prior analgesics & 0 & 0 \\
\hline ANTI_ANDROGENS & Binary & Prior Anti-Androgens & 0 & 0 \\
\hline GLUCOCORTICOID & Binary & Prior Glucocorticoids & 0 & 0 \\
\hline GONADOTROPIN & Binary & Prior Gomadotropin & 0 & 0 \\
\hline BISPHOSPHONATE & Binary & Prior Bisphosponate & 0 & 0 \\
\hline CORTICOSTEROID & Binary & Prior Corticosteroid & 0 & 0 \\
\hline IMIDAZOLE & Binary & Prior Imidazole & 0 & 0 \\
\hline ACE_INHIBITORS & Binary & Prior ACE Inhibitors & 0 & 0 \\
\hline BETA_BLOCKING & Binary & Prior Beta Blocking Agents & 0 & 0 \\
\hline HMG_COA_REDUCT & Binary & Prior HMG COA Reductase Inhibitors & 0 & 0 \\
\hline ESTROGENS & Binary & Prior Estrogens & 0 & 0 \\
\hline ANTI_ESTROGENS & Binary & Prior Anti-Estrogens & 0 & 0 \\
\hline CEREBACC & Binary & Cerebrovascular accident & 0 & 0 \\
\hline $\mathrm{CHF}$ & Binary & Congestive heart failure & 0 & 0 \\
\hline DVT & Binary & Deep venous thrombosis & 0 & 0 \\
\hline DIAB & Binary & Diabetes & 0 & 0 \\
\hline GASTREFL & Binary & Gastroesophageal reflux disease & 0 & 0 \\
\hline GIBLEED & Binary & Gastrointestinal bleed & 0 & 0 \\
\hline
\end{tabular}




\begin{tabular}{|c|c|c|c|c|}
\hline Variable name & Type & Description & $\begin{array}{l}\text { Missing } \\
\text { in training }\end{array}$ & $\begin{array}{l}\text { Missing in } \\
\text { validation }\end{array}$ \\
\hline $\mathrm{Ml}$ & Binary & Myocardial infarction & 0 & 0 \\
\hline PUD & Binary & Peptic ulcer disease & 0 & 0 \\
\hline PULMEMB & Binary & Pulmonary embolism & 0 & 0 \\
\hline PATHFRAC & Binary & Pathological bone fractures & 0 & 0 \\
\hline SPINCOMP & Binary & Spinal cord compression & 0 & 0 \\
\hline COPD & Binary & Chronic obstructive pulmonary disease & 0 & 0 \\
\hline MHBLOOD & Binary & Blood \& lymphatic system & 0 & 0 \\
\hline MHCARD & Binary & Cardiac disorders & 0 & 0 \\
\hline MHCONGEN & Binary & Congenital, familial \& genetic & 0 & 0 \\
\hline MHEAR & Binary & ear \& Labyrinth & 0 & 0 \\
\hline MHENDO & Binary & Endocrine disorders & 0 & 0 \\
\hline MHEYE & Binary & Eye disorders & 0 & 0 \\
\hline MHGASTRO & Binary & Gastrointestinal disorders & 0 & 0 \\
\hline MHGEN & Binary & Gen. disord \& admin site & 0 & 0 \\
\hline MHHEPATO & Binary & Hepatobiliary disorders & 0 & 0 \\
\hline MHIMMUNE & Binary & Immune system disorders & 0 & 0 \\
\hline MHINFECT & Binary & Infections \& infestations & 0 & 0 \\
\hline MHINJURY & Binary & Injury, poison \& procedural & 0 & 0 \\
\hline MHINVEST & Binary & Investigations & 0 & 0 \\
\hline MHMETAB & Binary & Metabolism \& nutrition & 0 & 0 \\
\hline MHMUSCLE & Binary & Musc./skeletal \& connect tissue & 0 & 0 \\
\hline MHNERV & Binary & Nervous system disorders & 0 & 0 \\
\hline MHPSYCH & Binary & Psychiatric disorders & 0 & 0 \\
\hline MHRENAL & Binary & Renal \& urinary disorders & 0 & 0 \\
\hline MHRESP & Binary & Resp., thoracic \& mediastinal & 0 & 0 \\
\hline MHSKIN & Binary & Skin \& subcutaneous tissue & 0 & 0 \\
\hline MHSOCIAL & Binary & Social circumstances & 0 & 0 \\
\hline MHSURG & Binary & Surgical \& medical procedures & 0 & 0 \\
\hline MHVASC & Binary & Vascular disorders & 0 & 0 \\
\hline $\mathrm{BMI}^{*}$ & Numerical & Baseline body mass index (kg/m2) & 10 & 1 \\
\hline $\mathrm{ALP}^{*}$ & Numerical & Alkaline phosphatase u/l & 5 & 2 \\
\hline ALT & Numerical & Alanine transaminase $u / l$ & 5 & 3 \\
\hline $\mathrm{AST}^{*}$ & Numerical & Aspartate aminotransferase $\mathrm{u} / \mathrm{l}$ & 13 & 3 \\
\hline CA & Numerical & Calcium mmol/l & 11 & 2 \\
\hline CREAT & Numerical & Creatinine umol/l & 3 & 2 \\
\hline $\mathrm{HB}^{*}$ & Numerical & Hemoglobin g/dl & 13 & 4 \\
\hline LDH & Numerical & Lactate dehydrogenase $u / l$ & 610 & 4 \\
\hline NEU & Numerical & Neutrophils $10^{9} / l$ & 21 & 4 \\
\hline PLT & Numerical & Platelet count $10^{9} / 1$ & 17 & 10 \\
\hline PSA & Numerical & Prostate specific antigen ng/ml & 11 & 10 \\
\hline TBILI & Numerical & Total bilirubin umol/l & 23 & 2 \\
\hline TESTO & Numerical & Testosterone nmol// & 855 & 2 \\
\hline WBC & Numerical & White blood cells $10^{9} / 1$ & 13 & 4 \\
\hline NA. & Numerical & Sodium mmol/l & 481 & 2 \\
\hline MG & Numerical & Magnesium mmol// & 510 & 2 \\
\hline PHOS & Numerical & Phosphorus mmol/l & 504 & 2 \\
\hline ALB $^{*}$ & Numerical & Albumin $\mathrm{g} / \mathrm{l}$ & 493 & 2 \\
\hline TPRO & Numerical & Total protein g/l & 504 & 2 \\
\hline
\end{tabular}


1. DREAM9.5 - Prostate Cancer DREAM Challenge. 2015 Publisher Full Text

2. Guinney J, Wang T, Laajala TD, et al.: Prediction of overall survival for patients with metastatic castration-resistant prostate cancer: development of a prognostic model through a crowdsourced challenge with open clinical trial data. Lancet Oncol. 2016.

Publisher Full Text

3. Halabi S, Lin CY, Kelly WK, et al.: Updated prognostic model for predicting overall survival in first-line chemotherapy for patients with metastatic castration-resistant prostate cancer. J Clin Oncol. 2014; 32(7): 671-7. PubMed Abstract | Publisher Full Text | Free Full Text

4. Scher HI, Jia X, Chi K, et al.: Randomized, open-label phase III trial of docetaxe plus high-dose calcitriol versus docetaxel plus prednisone for patients with castration-resistant prostate cancer. J Clin Oncol. 2011; 29(16): 2191-2198. PubMed Abstract | Publisher Full Text

5. Petrylak DP, Vogelzang NJ, Budnik N, et al:: Docetaxel and prednisone with or without lenalidomide in chemotherapy-naive patients with metastatic castration-resistant prostate cancer (MAINSAIL): a randomised, double-blind, placebo-controlled phase 3 trial. Lancet Oncol. 2015; 16(4): 417-425. PubMed Abstract | Publisher Full Text

6. Tannock IF, Fizazi K, Ivanov S, et al:: Aflibercept versus placebo in combination with docetaxel and prednisone for treatment of men with metastatic castration-resistant prostate cancer (VENICE): a phase 3, double-blind randomised trial. Lancet Oncol. 2013; 14(8): 760-768. PubMed Abstract | Publisher Full Text

7. Fizazi K, Higano CS, Nelson JB, et al: Phase III, randomized, placebo-controlled study of docetaxel in combination with zibotentan in patients with metastatic castration-resistant prostate cancer. J Clin Oncol. 2013; 31(14): 1740-7. PubMed Abstract | Publisher Full Text

8. White IR, Royston P: Imputing missing covariate values for the Cox model. Stat Med. 2009; 28(15): 1982-1998.

PubMed Abstract | Publisher Full Text | Free Full Text

9. Therneau TM, Grambsch PM: Modeling Survival Data: Extending the Cox Model. Springer, New York, 2000 Publisher Full Text

10. Therneau TM: A Package for Survival Analysis in S. Version 2.38. 2015 Reference Source

11. Wood SN: Generalized Additive Models: An Introduction with R. Chapman and Hall/CRC. 2006; 16.

Reference
12. Friedman J, Hastie T, Tibshirani R: Regularization Paths for Generalized Linear Models via Coordinate Descent. J Stat Softw. 2010; 33(1): 1-22. PubMed Abstract | Publisher Full Text | Free Full Text

13. Simon N, Friedman J, Hastie T, et al.: Regularization Paths for Cox's Proportiona Hazards Model via Coordinate Descent. J Stat Softw. 2011; 39(5): 1-13. PubMed Abstract | Publisher Full Text | Free Full Text

14. Meinshausen N, Bühlmann P: Stability selection. J R Stat Soc Series B Stat Methodol. 2010; 72(4): 417-473. Publisher Full Text

15. Friedman JH: Stochastic gradient boosting. Comput Stat Data Anal. 2002; 38(4): 367-378. Publisher Full Text

16. Chen $\mathrm{Y}$, Jia Z, Mercola $\mathrm{D}$, et al:: A gradient boosting algorithm for survival analysis via direct optimization of concordance index. Comput Math Methods Med. 2013; 2013: 873595 PubMed Abstract | Publisher Full Text | Free Full Text

17. Fork of the gbm R package.

Reference Source

18. Ridgeway G: gbm: Generalized Boosted Regression Models. R package version 1.6-3.2, 2012. Reference Source

19. Ishwaran $\mathrm{H}$, Kogalur UB, Blackstone $\mathrm{EH}$, et al.: Random survival forests. Ann Appl Stat. 2008; 2(3): 841-860. Publisher Full Text

20. Ishwaran H, Kogalur UB, Gorodeski EZ, et al.: High-dimensional variable selection for survival data. J Am Stat Assoc. 2010; 105(489): 205-217. Publisher Full Text

21. Blanche P, Dartigues JF, Jacqmin-Gadda H: Estimating and comparing timedependent areas under receiver operating characteristic curves for censored event times with competing risks. Stat Med. 2013; 32(30): 5381-5397. PubMed Abstract | Publisher Full Text

22. Hansen NR, Buchardt AS, Dunbar MN, et al:: Prostate Cancer DREAM Challenge submission and write-up by team KUStat. 2015 Publisher Full Text

23. Bühlmann P, van de Geer S: Statistics for high-dimensional data. Springer Series in Statistics. Springer, Heidelberg. 2011. Publisher Full Text

24. Mogensen SW, Petersen AH, Buchardt AS, et al.: ProstateDream: Prostate Dream code. Zenodo. 2016 


\section{Open Peer Review}

\section{Current Peer Review Status:}

\section{Version 1}

Reviewer Report 22 December 2016

https://doi.org/10.5256/f1000research.9071.r17680

(c) $2016 \mathrm{Hjelmborg} \mathrm{J.} \mathrm{This} \mathrm{is} \mathrm{an} \mathrm{open} \mathrm{access} \mathrm{peer} \mathrm{review} \mathrm{report} \mathrm{distributed} \mathrm{under} \mathrm{the} \mathrm{terms} \mathrm{of} \mathrm{the} \mathrm{Creative}$ Commons Attribution License, which permits unrestricted use, distribution, and reproduction in any medium, provided the original work is properly cited.

\section{Jacob von Bornemann Hjelmborg}

Epidemiology, Biostatistics and Biodemography Unit, University of Southern Denmark, Odense, Denmark

This paper deals with the very hard and important problem of cancer survival prognosis with focus on evaluation of methods and data usages. Common prognosis models are assessed in a full-scale analysis, that is, in combination with imputation techniques and variable selection methods.

The data stems from the prostate cancer DREAM challenge and the stage is set for achieving best prognosis performance in terms of the integrated AUC in case of cross-validation using the training data or for the validation dataset. The authors provide a thoroughly description of predictors and their mutual relationship.

Combined Methods: The missingness taxonomy, which sets the range for imputation, is chosen sufficiently exhaustive and is then combined with the modelling and variable selection approaches. The chosen stability selection is appropriate and interesting given the wide range of chosen models. Some of the models have implicit variable selection and comparisons are made.

Interestingly the debiased lasso is shown to perform rather poorly in the comparison scenario as well as the boosting methods that optimizes a concordance measure, but are low on the iAUCs, somewhat unexpected. Explaining why the methods differ in performance is not straightforward but the general purpose of comparison is fully met and very interesting claims are provided useful for developing prognostic survival models. The stability selection procedure is recommended as a reliable method for selecting prognostic variables.

The authors point at ad-hoc choices and "hand tuning" during the process, but manage to convey and address very well the great many choices to be made for survival prognosis in action.

There is a comprehensive explanation of study design, chosen methods, analysis, and results. The paper is clearly written and may in combination with the supplied material serve as a very good tutorial on the topic - the case study presented seems to have very general scope for application. 
Papers of this type are welcomed in general.

Misc:

A typo of AUC in abstract.

A specification of how mutual dependence of binary predictors is obtained.

Not important: the estimation of the iAUC was not to be found in the supplied material - maybe a closer look is needed.

Competing Interests: No competing interests were disclosed.

\section{I confirm that I have read this submission and believe that I have an appropriate level of expertise to confirm that it is of an acceptable scientific standard.}

Reviewer Report 30 November 2016

https://doi.org/10.5256/f1000research.9071.r17681

(C) 2016 Lemler S. This is an open access peer review report distributed under the terms of the Creative Commons Attribution License, which permits unrestricted use, distribution, and reproduction in any medium, provided the original work is properly cited.

\section{Sarah Lemler}

Laboratory of Mathematics in Interaction with Computer Science, Ecole Centrale Supélec, Châtenay-Malabry, France

The authors have participated to the Prostate Cancer DREAM challenge. They report in this paper the methodology developed to predict the survival of patients with prostate cancer. They detail different methods, combining imputation techniques to replace missing values in the dataset with selection and model fitting procedures to predict the survival. They compare these methods to each other using the integrated AUC and give their conclusion about the best method in terms of prediction.

My opinion on the paper is the following. First of all, the paper is very well written and pleasant to read. It is clear and understandable. The procedures and methodologies followed by the authors are well explained in a concise way. In addition, the different methods considered in the paper to deal with the Prostate Cancer data are interesting not only for the dataset of the challenge, but also for other types of data. The paper gives some methodology that can be applied to any other dataset to study the survival. Lastly, the comparison of those methods shows that the best procedure is not always the one that we thought, but depends on the objective. This paper shows for example that the random survival forest is better in prediction than the Lasso, which is good in selection. As a conclusion, I strongly approve the publication of this paper.

Some questions and little comments to improve the form:

1. Page 3, line 3: the verb "found" has to be removed.

2. Does the choice of the training, test and validation data influence the results? Would it be 
interesting to compare the results by changing these different samples?

3. Table 2: maybe, it would be easier for the reader to detect the differences of the age distribution in the four trials with the proportion by age class instead of the number of patients.

4. Figure 3 and paragraph 2: should we not remove the variables with lots of missing values when we do stability selection? How could you explain that the variable ALB with many missing values has been selected for example and does one really need to select this variable?

Competing Interests: No competing interests were disclosed.

I confirm that I have read this submission and believe that I have an appropriate level of expertise to confirm that it is of an acceptable scientific standard.

The benefits of publishing with F1000Research:

- Your article is published within days, with no editorial bias

- You can publish traditional articles, null/negative results, case reports, data notes and more

- The peer review process is transparent and collaborative

- Your article is indexed in PubMed after passing peer review

- Dedicated customer support at every stage

For pre-submission enquiries, contact research@f1000.com 